\title{
Surgical results of 23-gauge pars plana vitrectomy in adult traumatic retinal detachment
}

\author{
Dilek Yaşa*, Zeynep Alkın \\ Prof. Dr. N. Reşat Belger Beyoğlu Ophtalmology Training and Research Hospital
}

\begin{abstract}
To evaluate the anatomic and functional results of 23 gauge pars plana vitrectomy (PPV) in eyes with traumatic retinal detachment (RD), medical records of patients who had each undergone PPV surgery after traumatic RD diagnoses were retrospectively reviewed. Patients who had at least 6 months of follow-up were included in the study. Patients' clinical characteristics, preoperative and postoperative distance corrected visual acuities, anatomical success rates, and complications were analyzed.

Twenty-seven eyes from 27 patients were included in the study. Mean patient age was $48 \pm 17$ years, and $96 \%$ of the cases were male. Mean follow-up period was $8 \pm 3$ months. Seventeen $(63 \%)$ patients had closed and $10(37 \%)$ patients had open globe trauma. Five $(19 \%)$ patients had an intraocular foreign body. Mean time from trauma to PPV was $34 \pm 33$ days. Anatomical success was achieved in $19(70 \%)$ eyes. Functional success was achieved in $13(48 \%)$ eyes. Preoperative (baseline) visual acuity was the only factor associated with functional success.

Traumatic RD may result in severe visual loss in the majority of cases despite the advancements in PPV techniques. Functional success rate is higher in patients presenting better preoperative (baseline) visual acuity.
\end{abstract}

Key Words: Ocular trauma, open globe, close globe, intraocular foreign body, epidemiology, Turkey

\section{Introduction}

Ocular trauma is a leading cause of blindness, and traumatic retinal detachment (RD) accounts for approximately one-third of all RD cases $(1,2)$. Traumatic RD is associated with poor functional outcomes in traumatized eyes (3).

Pars plana vitrectomy (PPV) is the preferred surgical method for treatment of traumatic RD because it allows for management of associated posterior segment complications. It has improved surgical outcomes in severely traumatized eyes and may result in anatomical success in eyes that were previously deemed inoperable.

The functional and anatomical success rates after trauma surgery are closely related to the nature of the ocular trauma and associated complications (2). The incidence and nature of ocular trauma are different in different countries.

In this study, we evaluated the clinical characteristics and associated pathologies of patients who had a diagnosis of traumatic $\mathrm{RD}$ and who underwent $23 \mathrm{G}$ PPV surgery in a tertiary referral eye hospital in Turkey. We also investigated the factors that influenced final visual acuity and anatomical success rates.

\section{Material and Methods}

Medical records of patients with a diagnosis of traumatic RD who had undergone $23 \mathrm{G}$ PPV surgery during the period from January 2017 to January 2018 were retrospectively reviewed. This study followed the tenets of the Declaration of Helsinki and approval was obtained from the local ethics committee. Patients who are $\geq 18$ years old and who had at least 6 months of follow-up were included in the study. Age, gender, ocular trauma type (according to the Ocular Trauma Classification), associated pathologies, time between injury and PPV, surgical procedures, preoperative (baseline) distance corrected visual acuity (DCVA), and final DCVA and complications were recorded. Anatomical success was defined as total retinal attachment at the final visit, and functional success was defined as DCVA $\geq 5 / 200$. Visual acuity was assessed in each followup visit using an Early Treatment Diabetic Retinopathy Study chart. The association between functional/anatomical success and several factors was analyzed: 1.) type of injury; 2.) presence of intraocular foreign body (IOFB); 3.) time between injury and PPV; 4.) preoperative DCVA; 5.) presence of proliferative vitreoretinopathy (PVR) 
Table 1. Associated ocular pathologies

\begin{tabular}{lc}
\hline Ocular Pathology & $\mathrm{n} \mathrm{( \% )}$ \\
\hline Hyphema & $4(15 \%)$ \\
Cyclodialysis/Iridodialysis & $1(4 \%)$ \\
Traumatic Cataract & $7(26 \%)$ \\
Crystalline lens & $5(19 \%)$ \\
subluxation/dislocation & \\
Intravitreal hemorrhage & $8(30 \%)$ \\
Choroidal detachment & $3(11 \%)$ \\
PVR Grade B & $9(33 \%)$ \\
Endophthalmitis & $1(4 \%)$ \\
\hline
\end{tabular}

n: Number, PVR: Proliferative Vitreoretinopathy

$\geq$ Grade $\mathrm{B}$ according to the updated Retina Society Classification; and 5.) use of silicone oil as an internal tamponade.

Surgical Technique: All cases were operated on with standard 23-gauge vitrectomy techniques using Constellation Surgical Vitrectomy System (Alcon Laboratories Inc., Fort Worth, TX, USA). Phacoemulsification or pars plana lensectomy (PPL) was performed in eyes with lens opacity or subluxation/dislocation. A core vitrectomy was performed to remove media opacity, and posterior hyaloid was stripped from the retinal surface in eyes without posterior hyaloid separation. Perfluorocarbon liquids were used to attach the retina and retinal breaks were treated with photocoagulation delivered with endolaser. Relaxing retinotomies or retinectomies were performed in the presence of retinal shortening resulting from retinal incarceration or fibrous proliferation. A scleral buckle was placed when the anatomical status of the eye did not permit complete removal of the vitreous base. Air, Sulfur hexafluoride (SF6), perfluoropropane (C3F8), or silicone oil was used as an internal tamponade.

Statistical Analysis: Statistical analysis was carried out using SPSS 17.0 software (SPSS Inc., Chicago, IL, USA). Categorical variables were analyzed using $\chi 2$ and Fisher's exact test. Nonparametric variables were compared with the Mann Whitney U test. A two-tailed p-value $<0.05$ was considered statistically significant.

\section{Results}

Twenty-seven eyes from 27 patients were included in the study. Twenty-six (96\%) of the patients were male, and mean patient age was $48 \pm 17$ years (Range 22 to 79 years). Ten (37\%) patients had open globe trauma, and $17(63 \%)$ patients had closed globe trauma. $5(19 \%)$ patients had an intraocular foreign body (IOFB). When all cases were analyzed together, the mean time from injury to PPV was $34 \pm 33$ days (Range 4 to 120 days) The mean time between trauma and PPV was shorter in open globe trauma when compared to closed globe trauma ( $25 \pm 27$ days versus $40 \pm 37$ days). However, the difference was not statistically significant $\mathrm{p}<0.05$ by Mann Whitney $\mathrm{U}$ test). The mean follow-up time was $8 \pm 3$ months (Range 6 to 12 months). Associated pathologies are shown in Table 1. Relaxing retinectomies were performed in six $(22 \%)$ eyes. Silicone oil was used as an internal tamponade in $22(81 \%)$ eyes, sulfur hexafluoride was used in three $(11 \%)$ eyes, and octafluoropropane was used in two (7\%) eyes. Postoperative glaucoma developed in two (7\%) eyes, and intraocular pressure was under control with medical treatment in these eyes. Cumulative baseline and final visual acuity of the patients are shown in Figure 1. At the final visit, anatomical success was achieved in $19(70 \%)$ eyes, and functional success was achieved in 13 (48\%) eyes. In the postoperative period, two $(6 \%)$ patients lost light perception. These patients had PVR Grade C and chronic hypotony at the last follow-up visit. Patients with hand motion or better visual acuity were more likely to have functional success at the final visit (Table 2).

\section{Discussion}

In this study, we retrospectively analyzed data from patients with ocular trauma and RD who had undergone $23 \mathrm{G}$ PPV. In our study, males and male to female ratio were $96 \%$ and $26: 1$, respectively. It is known that ocular trauma is more common in males. Zhang et al. (4) reported a male-to-female ratio of 5:1 for ocular trauma from a tertiary referral hospital in China, and studies from European countries have reported ratios ranging from 5 to $6: 1$ for male-to-female ratios $(5,6)$. Although our study is in agreement with other studies in which the incidence of traumatic retinal detachment is higher in males, the male-to-female ratio in our study was much higher when compared to other studies described in the literature (7). This probably reflects cultural and social differences among countries.

RD does not always occur immediately after trauma. In our patients, the mean time from trauma to PPV was $34 \pm 33$ days but ranged from 4 to 120 days. There were four outliers who were operated on $\geq 90$ days after the trauma, and this increased the mean time from trauma to PPV. In fact, most of the patients underwent surgery in the

East J Med Volume:23, Number:4, October-December/2018 
Table 2. Factors associated with anatomical and functional success

\begin{tabular}{|c|c|c|c|c|c|}
\hline Factor & $\mathrm{n}$ & Anatomical success & $\mathrm{p}$ & Functional success & $\mathrm{p}$ \\
\hline \multicolumn{6}{|l|}{ Injury type } \\
\hline Open globe trauma & 10 & $6 / 10(60 \%)$ & \multirow[t]{2}{*}{0.415} & $4 / 10(40 \%)$ & \multirow[t]{2}{*}{0.695} \\
\hline Closed globe trauma & 17 & $13 / 17(77 \%)$ & & $9 / 17(53 \%)$ & \\
\hline \multicolumn{6}{|l|}{ Intraocular foreign b. } \\
\hline Present & 5 & $4 / 5(80 \%)$ & \multirow{2}{*}{1} & $4 / 5(80 \%)$ & \multirow{2}{*}{0.165} \\
\hline Absent & 22 & $15 / 22(68 \%)$ & & $9 / 22(41 \%)$ & \\
\hline \multicolumn{6}{|c|}{ Time from injury to PPV } \\
\hline$<2$ weeks & 9 & $6 / 9(67 \%)$ & \multirow{2}{*}{1} & $3 / 9(\% 33)$ & \multirow[t]{2}{*}{0.420} \\
\hline$>2$ weeks & 18 & $13 / 18(72 \%)$ & & $10 / 18(\% 56)$ & \\
\hline \multicolumn{6}{|l|}{ Presenting DCVA } \\
\hline$\geq$ Hand motion & 15 & $12 / 15(80 \%)$ & \multirow{2}{*}{0.398} & $11 / 15(73 \%)$ & \multirow{2}{*}{$0.006^{*}$} \\
\hline$<$ Hand motion & 12 & $7 / 12(58 \%)$ & & $2 / 12(17 \%)$ & \\
\hline \multicolumn{6}{|l|}{ PVR } \\
\hline$\geq$ Grade $B$ & 9 & $8 / 9(89 \%)$ & \multirow{2}{*}{0.201} & $4 / 9(44 \%)$ & \multirow{2}{*}{1} \\
\hline$<$ Grade $\mathrm{B}$ & 18 & $11 / 18(61 \%)$ & & $9 / 18(50 \%)$ & \\
\hline \multicolumn{6}{|l|}{ Silicone oil use } \\
\hline Yes & 22 & $14 / 22(64 \%)$ & \multirow[t]{2}{*}{0.280} & $12 / 22(55 \%)$ & \multirow[t]{2}{*}{0.326} \\
\hline No & 5 & $5 / 5(100 \%)$ & & $1 / 5(20 \%)$ & \\
\hline \multicolumn{6}{|c|}{ Intravitreal Hemorrhage } \\
\hline Present & 8 & $6 / 8(75 \%)$ & \multirow[t]{2}{*}{1} & $3 / 8(38 \%)$ & \multirow[t]{2}{*}{0.678} \\
\hline Absent & 19 & $13 / 19(68 \%)$ & & $10 / 19(53 \%)$ & \\
\hline \multicolumn{6}{|c|}{ Crystalline Lens damage } \\
\hline Present & 12 & $7 / 12(58 \%)$ & \multirow[t]{2}{*}{0.398} & $4 / 12(33 \%)$ & \multirow[t]{2}{*}{0.252} \\
\hline Absent & 15 & $12 / 15(80 \%)$ & & $9 / 15(60 \%)$ & \\
\hline
\end{tabular}

PPV: Pars plana vitrectomy, DCVA: Distance corrected visual acuity, PVR: Proliferative vitreoretinopathy according to the updated Retina Society Classification, RD: Retinal detachment

*: Fisher's exact test, two-tailed $\mathrm{p}$ value

first month after the initial trauma. Half of them underwent RD surgery on or before 22 days, and $67 \%$ underwent surgery in the first month. Stryjewski et al. (8) reported that in a series of 893 open globe injuries, $255(29 \%)$ were ultimately diagnosed with $\mathrm{RD}$, and $72 \%$ of these were detected one month after injury. In our cases, $80 \%$ of open trauma RD cases underwent surgery in the first month after injury; this result was very similar to that found in the larger series. Nowomiejska et al. (7) reported their results concerning traumatic RD cases; in their series, the mean time from injury to PPV was 67 days, 22 days in open trauma, and 102 days in closed trauma. Although RD may occur earlier in open globe trauma, we believe that the higher time interval between injury and PPV in closed globe trauma is at least partially a result of delayed diagnosis in these eyes.

Anatomical success rate was $70 \%$, and functional success rate was $48 \%$ in our patients. Phthisis bulbi developed in two eyes in this study and has been reported in up to $8 \%$ of cases in some series (9). As it would be expected, functional outcomes were worse when compared to anatomical outcomes because associated pathologies limit visual gains in most of the cases. Our findings were comparable to other series reported in the literature. Nasheed et al. [9] reported that $50 \%$ of the patients who have undergone PPV for RD after open globe trauma have a visual acuity of $5 / 200$ or better. Nowomiejska et al. (7) reported visual acuity of $5 / 200$ or better in $66 \%$ after PPV in a group of open and closed globe trauma and $\mathrm{RD}$ patients.

The patients with traumatic RDs show a high diversity of clinical profiles because the clinical profile depends on the nature of the injury. This diversity of clinical findings has led several investigators to study the factors that influence final visual acuity and anatomical success. In our patient group associated posterior segment 


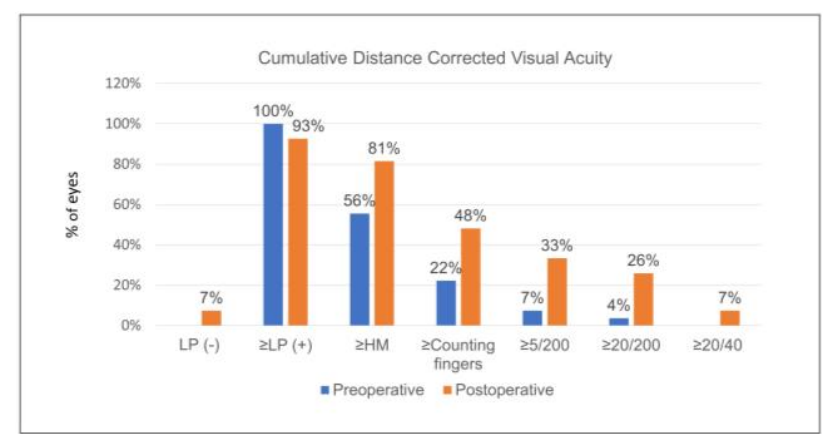

Fig. 1. Cumulative distance-corrected visual acuity

pathologies were intravitreal hemorrhage, choroidal detachment, and endophthalmitis. These are well known complications of severe ocular trauma and our results are comparable with the literature (7-11). Nowomiejska $\mathrm{K}$ et al (7) reported vitreous hemorrhage in $17 \%$ of patients who had traumatic RD. Unver et al [11] reported vitreous hemorrhage and choroidal detachment in 37\% and $11 \%$ of the eyes, respectively. In line with our study, these posterior segment pathologies were not found to be significant predictors of final visual acuity (7-11). In our patient group, associated anterior segment complications were traumatic cataract, lens dislocation/subluxation, hyphema and Iridodialysis / cyclodialysis. In agreement with the literature, we found that none of these associated findings were associated with the final visual acuity $(11,12)$.

The literature supports that better visual acuity at presentation predicts a better final visual acuity when compared to those with a poor visual acuity at presentation (baseline) (7-10,11). However, the definition of a good prognosis or categorization of patients varies between studies. Rouberol et al. [10] reported that good final visual prognosis $(\geq 20 / 40)$ was significantly associated with an initial visual acuity $>20 / 200$. In our patient group, $93 \%$ of the patients had visual acuity of light perception (LP), hand motion (HM), or counting fingers $(\mathrm{CF})$ at presentation (Table 2 and Figure 1). Thus, we categorized visual acuity accordingly and found that those with a hand motion or better visual acuity had better functional outcomes at the final visit.

In our study, functional and anatomical results were similar in open and closed ocular injuries. Rouberol et al. (10) compared final RD prognoses that were secondary to open or closed ocular injuries in 50 consecutive patients, and they found that anatomical and functional prognosis was similar in the two groups. In a group of 41 patients, Nowomiejska et al. (7) found that visual acuity was significantly improved in eyes with
IOFB. They also reported that postoperative visual acuity was significantly better in eyes without PVR at presentation. In our patients, although both anatomical (80\% versus $68 \%$ ) and functional $(80 \%$ versus $41 \%$ ) success rates were better in eyes with IOFB, the differences were not statistically significant. Thirty-three percent of our patients had PVR at presentation, and all of them had PVR Grade B. However, the presence of PVR Grade $B$ at presentation did not have a significantly adverse effect on functional or anatomic outcomes. These differences may be due to heterogeneity of trauma patients and other clinical differences between the patient groups in these studies or the slightly lower number of patients in our study.

The limitation of this study was the retrospective nature and diversity of the clinical features of the included patients. However, the variability of clinical features is an inherent property of traumatic patients, and thus, an individualized approach should be used.

In conclusion, we evaluated the clinical characteristics of traumatic RD patients in addition to anatomical and functional success rates of $23 \mathrm{G}$ PPV in these cases. Despite advances in surgical techniques, anatomical and functional outcomes, especially, are still not satisfactory in traumatic RD cases as only about half of the patients achieve a final visual acuity of $5 / 200$ or better. In this study, preoperative (baseline) visual acuity was the only factor associated with functional success rates (described as visual acuity of 5/200 or better).

\section{References}

1. Negrel AD, Thylefors B. The global impact of eye injuries. Ophthalmic Epidemiology 1998; 5: 143-169.

2. Balles MW. Traumatic retinopathy. In: Albert DM, Jakobiec FA (Eds). Principles and Practice of Ophthalmology. Philadelphia: WB Saunders 1994; 1029-1031.

3. Kuhn F, Maisiak R, Mann L, Mester V, Morris $\mathrm{R}$, Witherspoon CD. The Ocular Trauma Score (OTS). Ophthalmology Clinics of North America 2002; 15: 163-165.

4. Zhang X, Liu Y, Ji X, Zou Y. A Retrospective Study on Clinical Features and Visual Outcome of Patients Hospitalized for Ocular Trauma in Cangzhou, China. Journal of Ophthalmology 2017; 7694913.

5. Cillino S, Casuccio A, Di Pace F, Pillitteri F, Cillino G. A five-year retrospective study of the epidemiological characteristics and visual 
outcomes of patients hospitalized for ocular trauma in a Mediterranean area. BMC Ophthalmology 2008; 8: 6.

6. Morris DS, Willis S, Minassian D, Foot B, Desai P, and Mac Ewen CJ. The incidence of serious eye injury in Scotland: a prospective study. Eye 2014; 28: 34-40.

7. Nowomiejska K, Choragiewicz T, Borowicz $\mathrm{D}$, et al. Surgical management of traumatic retinal detachment with primary vitrectomy in adult patients. Journal of Ophthalmology 2017; 5084319.

8. Stryjewski TP, Andreoli CM, Eliott D. Retinal detachment after open globe injury. Ophthalmology 2014; 121: 327-333.

9. Nashed A, Saikia P, Herrmann W.A, Gabel VP, Helbig H, Hillenkamp J. The outcome of early surgical repair with vitrectomy and silicone oil in open-globe injuries with retinal detachment. American Journal of Ophthalmology 2011; 151: 522-528.

10. Rouberol F, Denis P, Romanet JP, Chiquet C. Comparative study of 50 early-or late-onset retinal detachments after open or closed globe injury. Retina 2011; 31: 1143-1149.

11. Unver YB, Acar N, Kapran Z, Altan T. Prognostic factors in severely traumatized eyes with posterior segment involvement. Turkish Journal of Trauma \& Emergency Surgery 2009; 15: 271-276.

12. De Juan E Jr, Strenberg P Jr, Michels RG. Penetrating ocular injuries. Types of injuries and visual results. Ophthalmology 1983; 90: 1318-1322. 